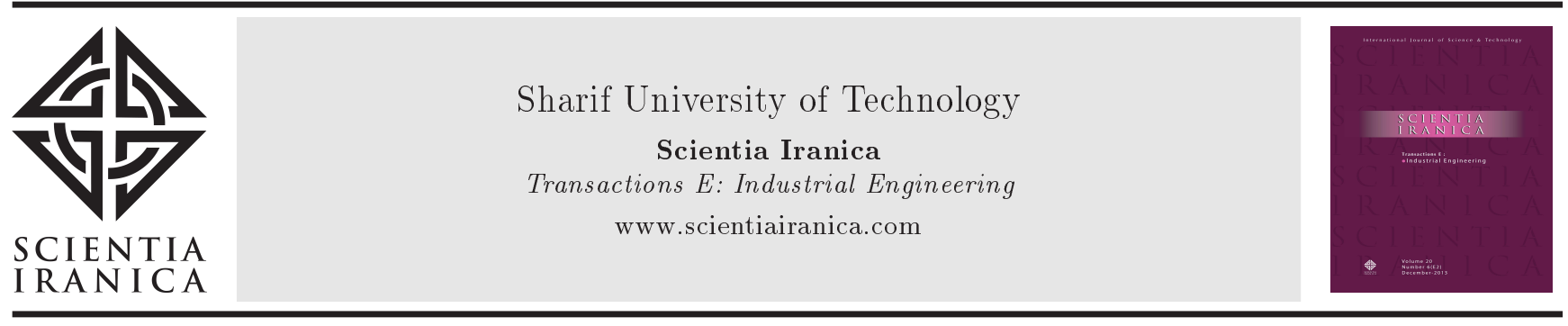

\title{
A method for multi-attribute group decision-making with triangular intuitionistic fuzzy numbers application to trustworthy service selection
}

\author{
S.-P. Wan ${ }^{\mathrm{a}}$ and J. Xu ${ }^{\mathrm{b}, *}$ \\ a. College of Information Technology, Jiangxi University of Finance and Economics, Nanchang 330013, China. \\ b. College of Modern Economics \& Management, Jiangxi University of Finance and Economics, Nanchang 330013, China.
}

Received 26 February 2015; received in revised form 23 May 2015; accepted 2 May 2016

\author{
KEYWORDS \\ Trustworthy service \\ selection; \\ Multi-attribute group \\ decision making; \\ Triangular \\ intuitionistic fuzzy \\ numbers; \\ Mean-index; \\ Variance-index; \\ Relative closeness \\ coefficient.
}

\begin{abstract}
The trustworthy service selection is a typical Multi-Attribute Group DecisionMaking (MAGDM) problem. The aim of this paper is to develop a novel method for MAGDM with Triangular Intuitionistic Fuzzy Numbers (TIFNs) and apply it to the trustworthy service selection problem. Firstly, we define the mean-index, variance-index, and standard deviation of TIFN. Moreover, a new distance measure of TIFNs is proposed, and the corresponding proofs are given. Based on these concepts of mean-index and standard deviation, a ranking method for TIFNs is developed considering the risk preference of Decision Maker (DM). Further, according to the crisp relative closeness coefficient matrix with respect to the normalized TIFNs decision matrix, we use entropy measure to obtain attribute weights. The DMs' weights are calculated by the similarity between the individual and the average decisions. Then, a decision procedure is described to solve the MAGDM under triangular intuitionistic fuzzy environment. Finally, a real trustworthy service selection example is analyzed to verify the practicality and effectiveness of the developed method.

(C) 2017 Sharif University of Technology. All rights reserved.
\end{abstract}

\section{Introduction}

In the ever-increasingly business competitiveness, electronic commerce has become a main stream practice in global business operations. Online service trading usually takes place between parties who are autonomous in an environment where the buyer often does not have enough information about the seller and goods. Many scholars think that trust is a prerequisite to successful trading. Therefore, it is very important that the buyers can identify the most trustworthy seller. The real-life trustworthy service evaluation often involves multiple different types of attributes (or indices, factors). It is not easy for Decision-Makers (DM) or experts to

*. Corresponding author. Tel.: +86-13755776992;

E-mail address: xujun1028@126.com (J.Xu) give the precise numerical assessment information. Therefore, trustworthy service selection problems may be ascribed to a kind of fuzzy Multi-Attribute Group Decision-Making (MAGDM) problems.

The concept of fuzzy numbers, introduced by Dubois and Prade [1], has received a great deal of attention due to its capability of representing fuzzy opinions [2]. Various methods have been developed to solve Multi-Attribute Decision-Making (MADM) problems with fuzzy numbers [3]. Intuitionistic Fuzzy Number (IFN) [4-6] is a special case of Intuitionistic Fuzzy Set (IFS) [7-10]. As an extension of fuzzy number, IFN may express the imprecise or uncertain decision information more abundantly and flexibly compared to the fuzzy set [11]. Recently, the Triangular Intuitionistic Fuzzy (TIF) Numbers (TIFNs) [1227], as a kind of typical IFNs, have attracted more and 
more considerations through a large amount of literature. A number of achievements on TIFNs have been proposed. Roughly, these achievements may be divided into two types: ranking methods of TIFNs and their applications to decision making problems [13-21] and aggregation operators of TIFNs and their applications to decision making problems [22-27], which are briefly reviewed in the following, respectively.

The first type is the ranking of TIFNs. Li [13] presented a ranking method on the basis of the concept of a ratio of the value index to the ambiguity index and applied it to MADM problems in which the ratings of alternatives on attributes are expressed with TIFNs. Li et al. [14] defined the value-index and ambiguityindex based ranking method for TIFNs. Zhang et al. [15] defined the distance measure of TIFNs and gave a ranking method based on TOPSIS. Wan et al. [1618] introduced the concepts of the weighted possibility mean, variance, and covariance of TIFNs, and they proposed some ranking methods of TIFNs and applied them to deal with MADM problems. Based on the possibility method, Wan and Dong [19] developed a method for MAGDM based on TIFNs with incomplete preference information. In addition to other methods, Nan et al. [20] investigated the ranking order relations of TIFNs, which are applied to matrix games with payoffs of TIFNs.

The second type is the aggregation operators of TIFN. Zhang and Liu [21] defined the concepts of TIFN in which the membership and the non-membership degrees are denoted by triangular fuzzy number. Then, the weighted geometric averaging operator and the weighted arithmetic average operator are presented and used for the decision-making area. Robinson and EC [22] investigated Triangular Intuitionistic Fuzzy Ordered Weighted Averaging (TIFOWA) operator and the Triangular Intuitionistic Fuzzy Hybrid Aggregation (TIFHA) operator. Chen and $\mathrm{Li}$ [23] developed a new distance measure between two TIFNs to aid in determining attribute weights, and they presented the Weighted Arithmetic Averaging operator on TIFNs (TIFN-WAA), and then proposed a dynamic MADM model with TIFNs. Wang et al. [24] proposed new arithmetic operations and logic operators for TIFNs and applied them to fault analysis of a printed circuit board assembly system. $\mathrm{Yu}$ and $\mathrm{Xu}$ [25] defined the concepts of Intuitionistic Multiplicative Triangular Fuzzy (IMTF) set and intuitionistic multiplicative triangular fuzzy number, and then discussed their operational laws and some desirable properties. Based on the operational laws, they developed a series of aggregation operators for IMTF information. Combining the fuzzy measure and Choquet integral, Wan and Dong [26] defined the TIF Choquet integral aggregation operator and investigated some desirable properties for this operator.
Based on the above, the existing research studies have mainly focused on ranking method and aggregation operators of TIFNs. The aforementioned methods are very appropriate for solving the MADM and MAGDM problems with TIFNs. However, some limitations of the above methods are listed as follows:

(i) The existing ranking methods of TIFNs [14,15] do not take into account the risk preference of DM. In fact, the risk preference of DM may affect the final ranking order of fuzzy numbers [3]. Thus, it is necessary to capture the DM's risk preference during the process of ranking TIFNs;

(ii) There is no sufficient amount of literature data on the method for determining DMs' weights and attribute weights under TIFN environments. Wan et al. [27] proposed an extended VIKOR method for MAGDM in which the attributes values are TIFNs, and the attribute weights and DM weights are completely unknown, but the decision information may be lost in the process of calculation.

In actual trustworthy service evaluation, the product quality of a seller can be assessed by a TIFN ((3.1, $4.2,9.2) ; 0.6,0.1)$, which means that the minimum value of product quality is 3.1 , the maximum value is 9.2 , and the most possible value is 4.2. Meanwhile, the maximum membership degree for the most possible value is 0.6 , the minimum non-membership degree is 0.1 , and the indeterminacy is 0.1 . Moreover, it is very difficult for DM to accurately give the attribute weights due to various subjective and objective reasons. Thus, the trustworthy service selection is a typical MAGDM problem with TIFNs and incomplete preference information.

To overcome the aforementioned limitations, in this paper, we devote to solving the following four issues:

1. Define a new distance measure for TIFNs;

2. Develop a novel ranking method for TIFNs considering the risk preferences of DMs;

3. Determine the attribute weights using the entropy measure;

4. Calculate the DMs' weights employing the similarity between the individual decisions and the average decisions. Thereby, a new method is proposed for solving MAGDM with TIFNs and applied to trustworthy service selection.

The rest of this paper is organized as follows. Section 2 introduces basic concepts and definitions of TIFNs, and a new distance measure for TIFNs is defined. Section 3 gives the definitions of mean-index, variance-index as well as standard deviation of TIFNs. Hereby, a new ranking method of TIFNs is developed. 
Section 4 describes the MAGDM problems with TIFNs, determines the attribute weights and DMs weights, and then proposes the corresponding decision procedure. A trustworthy service selection example and a comparison analysis are given in Section 5. Finally, we conclude the paper in Section 6 .

\section{Basic concepts of triangular intuitionistic fuzzy numbers}

\subsection{The definition and operations of TIFNs}

In this section, the concepts and operations of TIFNs are introduced as follows.

Definition 1 [13]. A TIFN $\tilde{a}=\left((a, b, c) ; u_{\tilde{a}}, v_{\tilde{a}}\right)$ is a special IFS on real number set, $R$, whose membership and non-membership functions are, respectively, defined as follows and depicted in Figure 1:

$$
\mu_{\tilde{a}}(x)= \begin{cases}(x-a) u_{\tilde{a}} /(b-a) & \text { if } a \leq x<b \\ u_{\tilde{a}} & \text { if } x=b \\ (c-x) u_{\tilde{a}} /(c-b) & \text { if } b<x \leq c \\ 0 & \text { otherwise }\end{cases}
$$

and:

$$
v_{\tilde{a}}(x)= \begin{cases}{\left[b-x+\nu_{\tilde{a}}(x-a)\right] /(b-a)} & \text { if } a \leq x<b \\ \nu_{\tilde{a}} & \text { if } x=b \\ {\left[x-b+\nu_{\tilde{a}}(c-x)\right] /(c-b)} & \text { if } b<x \leq c \\ 1 & \text { otherwise }\end{cases}
$$

where values $u_{\tilde{a}}$ and $\nu_{\tilde{a}}$ represent the maximum degree of membership and the minimum degree of nonmembership, respectively, such that they satisfy the following conditions: $0 \leq u_{\tilde{a}} \leq 1,0 \leq \nu_{\tilde{a}} \leq 1$, $0 \leq u_{\tilde{a}}+\nu_{\tilde{a}} \leq 1$. Let $\pi_{\tilde{a}}(x)=1-\mu_{\tilde{a}}(x)-v_{\tilde{a}}(x)$ which is called as IF index of element $x$ in $\tilde{a}$. It is the degree of indeterminacy of element $x$ in $\tilde{a}$.

TIFN $\tilde{a}=\left((a, b, c) ; u_{\tilde{a}}, \nu_{\tilde{a}}\right)$ may describe an illknown quantity, which is approximately equal to $b$.

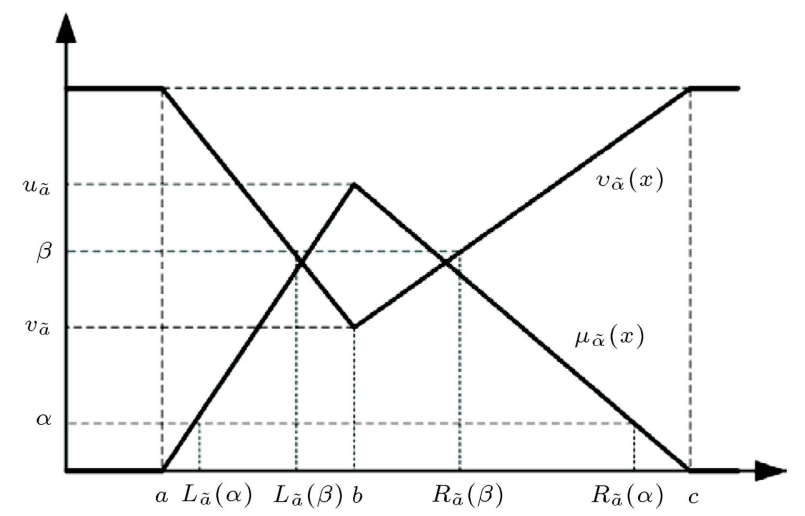

Figure 1. $a$-cut set of membership function and $\beta$-cut set of membership function.
That is to say, the most likely value is $b$ with degree $u_{\tilde{a}}$ of membership and degree $\nu_{\tilde{a}}$ of non-membership.

It is easy to see that $\mu_{\tilde{a}}(x)+v_{\tilde{a}}(x)=1$ for any $x \in R$ if $u_{\tilde{a}}=1$ and $\nu_{\tilde{a}}=0$. Hence, TIFN $\tilde{a}=\left((a, b, c) ; u_{\tilde{a}}, \nu_{\tilde{a}}\right)$ degenerates to $\tilde{a}=((a, b, c) ; 1,0)$, which is just about a TFN. Therefore, the concept of the TIFN is a generalization of that of the TFN.

Definition 2 [13]. Let $\tilde{a}_{1}=\left(\left(a_{1}, b_{1}, c_{1}\right) ; u_{\tilde{a}_{1}}, \nu_{\tilde{a}_{1}}\right)$ and $\tilde{a}_{2}=\left(\left(a_{2}, b_{2}, c_{2}\right) ; u_{\tilde{a}_{2}}, \nu_{\tilde{a}_{2}}\right)$ be two TIFNs, and $\lambda$ be a real number. Some arithmetical operations are defined as follows:

1. $\tilde{a}_{1}+\tilde{a}_{2}=\left(\left(a_{1}+a_{2}, b_{1}+b_{2}, c_{1}+c_{2}\right) ; \min \left\{u_{\tilde{a}_{1}}, u_{\tilde{a}_{2}}\right\}\right.$, $\left.\max \left\{\nu_{\tilde{a}_{1}}, \nu_{\tilde{a}_{2}}\right\}\right)$

2. $\tilde{a}_{1}-\tilde{a}_{2}=\left(\left(a_{1}-a_{2}, b_{1}-b_{2}, c_{1}-c_{2}\right) ; \min \left\{u_{\tilde{a}_{1}}, u_{\tilde{a}_{2}}\right\}\right.$, $\left.\max \left\{\nu_{\tilde{a}_{1}}, \nu_{\tilde{a}_{2}}\right\}\right)$

3. $\lambda \tilde{a}_{1}= \begin{cases}\left(\left(\lambda a_{1}, \lambda b_{1}, \lambda c_{1}\right) ; u_{\tilde{a}_{1}}, \nu_{\tilde{a}_{1}}\right) & \text { if } \lambda>0 \\ \left(\left(\lambda c_{1}, \lambda b_{1}, \lambda a_{1}\right) ; u_{\tilde{a}_{1}}, \nu_{\tilde{a}_{1}}\right) & \text { if } \lambda<0\end{cases}$

\subsection{Cut sets of TIFNs}

Definition 3 [12]. $(\alpha, \beta)$-cut set of TIFN $\tilde{a}=$ $\left((a, b, c) ; u_{\tilde{a}}, \nu_{\tilde{a}}\right)$ is a crisp subset of $R$, which is defined as $\tilde{a}_{\alpha, \beta}=\left\{x \mid \mu_{\tilde{a}}(x) \geq \alpha, v_{\tilde{a}}(x) \geq \beta\right\}$, where $0 \leq \alpha \leq u_{\tilde{a}}$, $\nu_{\tilde{a}} \leq \beta \leq 1$, and $0 \leq \alpha+\beta \leq 1$.

Definition 4 [12]. $\alpha$-cut set of TIFN $\tilde{a}=$ $\left((a, b, c) ; u_{\tilde{a}}, \nu_{\tilde{a}}\right)$ is a crisp subset of $R$, which is defined as $\tilde{a}_{\alpha}=\left\{x \mid \mu_{\tilde{a}}(x) \geq \alpha\right\}$, where $0 \leq \alpha \leq u_{\tilde{a}}$. Denoted by $\tilde{a}_{\alpha}=\left[L_{\tilde{a}}(\alpha), R_{\tilde{a}}(\alpha)\right]$, it can be calculated as follows:

$$
\left[L_{\tilde{a}}(\alpha), R_{\tilde{a}}(\alpha)\right]=\left[a+\frac{\alpha(b-a)}{u_{\tilde{a}}}, c-\frac{\alpha(c-b)}{u_{\tilde{a}}}\right] .
$$

Definition 5 [12]. $\beta$-cut set of TIFN $\tilde{a}=$ $\left((a, b, c) ; u_{\tilde{a}}, \nu_{\tilde{a}}\right)$ is a crisp subset of, which is defined as $\tilde{a}_{\beta}=\left\{x \mid v_{\tilde{a}}(x) \leq \beta\right\}$, where $\nu_{\tilde{a}} \leq \beta \leq 1$. Denoted by $\tilde{a}_{\beta}=\left[L_{\tilde{a}}(\beta), R_{\tilde{a}}(\beta)\right]$, it can be calculated as follows:

$$
\begin{aligned}
& {\left[L_{\tilde{a}}(\beta), R_{\tilde{a}}(\beta)\right]} \\
& \quad=\left[\frac{(1-\beta) b+\left(\beta-\nu_{\tilde{a}}\right) a}{1-\nu_{\tilde{a}}}, \frac{(1-\beta) b+\left(\beta-\nu_{\tilde{a}}\right) c}{1-\nu_{\tilde{a}}}\right] .
\end{aligned}
$$

\subsection{A new distance measure of TIFNs}

To deal with attribute value with TIFNs, several distance measures between TIFNs have been proposed and researched in recent years. Based on the Hausdorff distance proposed by Grzegorzewski [28], Wan et al. [27] proposed the following two distance measures between two TIFNs:

The Hamming distance:

1. $d_{h}\left(\tilde{a}_{1}, \tilde{a}_{2}\right)=\frac{1}{3}\left(\left|a_{1}-a_{2}\right|+\left|b_{1}-b_{2}\right|+\left|c_{1}-c_{2}\right|\right)+$ $\max \left(\left|u_{\tilde{a}_{1}}-u_{\tilde{a}_{2}}\right|,\left|\nu_{\tilde{a}_{1}}-\nu_{\tilde{a}_{2}}\right|\right)$; 
The Euclidean distance:

$$
\begin{array}{ll}
\text { 2. } & d_{e}\left(\tilde{a}_{1}, \tilde{a}_{2}\right)=\sqrt{\frac{1}{3}}\left[\left(a_{1}-a_{2}\right)^{2}+\left(b_{1}-b_{2}\right)^{2}+\left(c_{1}-\right.\right. \\
& \left.c_{2}\right)^{2}+\max \left(\left(u_{\tilde{a}_{1}}-u_{\tilde{a}_{2}}\right)^{2},\left(\nu_{\tilde{a}_{1}}-\nu_{\tilde{a}_{2}}\right)^{2}\right]^{1 / 2} .
\end{array}
$$

But, these above distance measures have some limitations.

Example 1. Consider the three TIFNs: $\tilde{a}_{1}=$ $((0.1,0.5,0.8) ; 1,0), \tilde{a}_{2}=((0.1,0.5,0.8) ; 0,1)$, and $\tilde{a}_{3}=$ $((0.1,0.5,0.8) ; 0,0)$. Obviously, the difference between $\tilde{a}_{1}$ and $\tilde{a}_{2}$ is larger than the difference between $\tilde{a}_{1}$ and $\tilde{a}_{3}$. However, according to the distance measures defined by Wan et al. [27], we have $d_{h}\left(\tilde{a}_{1}, \tilde{a}_{2}\right)=$ $d_{h}\left(\tilde{a}_{1}, \tilde{a}_{3}\right)$ and $d_{e}\left(\tilde{a}_{1}, \tilde{a}_{2}\right)=d_{e}\left(\tilde{a}_{1}, \tilde{a}_{3}\right)$, which are not reasonable.

Wan and Dong [26] defined a new hamming distance between two TIFNs:

$$
\begin{aligned}
& d_{h 1}\left(\tilde{a}_{1}, \tilde{a}_{2}\right)=\frac{1}{6}\left[\left|\left(1+u_{\tilde{a}_{1}}-\nu_{\tilde{a}_{1}}\right) a_{1}-\left(1+u_{\tilde{a}_{2}}-\nu_{\tilde{a}_{2}}\right) a_{2}\right|\right. \\
& \quad+\left|\left(1+u_{\tilde{a}_{1}}-\nu_{\tilde{a}_{1}}\right) b_{1}-\left(1+u_{\tilde{a}_{2}}-\nu_{\tilde{a}_{2}}\right) b_{2}\right| \\
& \left.\quad+\left|\left(1+u_{\tilde{a}_{1}}-\nu_{\tilde{a}_{1}}\right) c_{1}-\left(1+u_{\tilde{a}_{2}}-\nu_{\tilde{a}_{2}}\right) c_{2}\right|\right] .
\end{aligned}
$$

Although it can differentiate the above three TIFNs, it also has some disadvantages. If the maximum membership degree and the minimum nonmembership degree of some TIFNs are equal, then the distances between them are the same.

Example 2. Consider the three TIFNs: $\tilde{a}_{4}=$ $((0.1,0.5,0.8) ; 0.1,0.1), \quad \tilde{a}_{5}=((0.1,0.5,0.8) ; 0.3,0.3)$, and $\tilde{a}_{6}=((0.1,0.5,0.8) ; 0.5,0.5)$. By using the distance measure defined by Wan and Dong [26], we have $d_{h 1}\left(\tilde{a}_{4}, \tilde{a}_{6}\right)=d_{h 1}\left(\tilde{a}_{4}, \tilde{a}_{5}\right)=d_{h 1}\left(\tilde{a}_{6}, \tilde{a}_{5}\right)$. Obviously, the distance method also has shortcomings.

To overcome the dilemma of the above distance measures, we provide a new distance measure based on the method of Wang and Xin [29].

Definition 6. Let $\tilde{a}_{1}=\left(\left(a_{1}, b_{1}, c_{1}\right) ; u_{\tilde{a}_{1}}, \nu_{\tilde{a}_{1}}\right)$ and $\tilde{a}_{2}=\left(\left(a_{2}, b_{2}, c_{2}\right) ; u_{\tilde{a}_{2}}, \nu_{\tilde{a}_{2}}\right)$ be two TIFNs. The distance between $\tilde{a}_{1}$ and $\tilde{a}_{2}$ is defined as follows:

$$
\begin{aligned}
d\left(\tilde{a}_{1}, \tilde{a}_{2}\right)= & \frac{1}{6}\left(\left|a_{1}-a_{2}\right|+\left|b_{1}-b_{2}\right|+\left|c_{1}-c_{2}\right|\right) \\
& +\frac{1}{8}\left(\left|u_{\tilde{a}_{1}}-u_{\tilde{a}_{2}}\right|+\left|\nu_{\tilde{a}_{1}}-\nu_{\tilde{a}_{2}}\right|\right) \\
& +\frac{1}{4} \max \left(\left|u_{\tilde{a}_{1}}-u_{\tilde{a}_{2}}\right|,\left|\nu_{\tilde{a}_{1}}-\nu_{\tilde{a}_{2}}\right|\right) .
\end{aligned}
$$

If $u_{\tilde{a}_{i}}=1$ and $\nu_{\tilde{a}_{i}}=0$, then the TIFNs in Definition 1 reduce to Triangular Fuzzy Numbers (TFNs). Moreover, Eq. (5) reduces to Hamming distance of TFNs.
Theorem 1. The above distance, $d\left(\tilde{a}_{1}, \tilde{a}_{2}\right)$, satisfies the following properties:

(i) $0 \leq d\left(\tilde{a}_{1}, \tilde{a}_{2}\right) \leq 1$;

(ii) $d\left(\tilde{a}_{1}, \tilde{a}_{2}\right)=0$ if and only if $\tilde{a}_{1}=\tilde{a}_{2}$;

(iii) $d\left(\tilde{a}_{1}, \tilde{a}_{2}\right)=d\left(\tilde{a}_{2}, \tilde{a}_{1}\right)$;

(iv) If $\tilde{a}_{3}=\left(\left(a_{3}, b_{3}, c_{3}\right) ; u_{\tilde{a}_{3}}, \nu_{\tilde{a}_{3}}\right)$ is any TIFN, then $d\left(\tilde{a}_{1}, \tilde{a}_{3}\right) \leq d\left(\tilde{a}_{1}, \tilde{a}_{2}\right)+d\left(\tilde{a}_{2}, \tilde{a}_{3}\right)$.

Proof. Obviously, the proposed distance measure meets (i)-(iii) of Theorem 1 . We need only to prove (iv).

It is easy to see that:

$$
\left|u_{\tilde{a}_{1}}-u_{\tilde{a}_{3}}\right| \leq\left|u_{\tilde{a}_{1}}-u_{\tilde{a}_{2}}\right|+\left|u_{\tilde{a}_{2}}-u_{\tilde{a}_{3}}\right|,
$$

and:

$$
\left|\nu_{\tilde{a}_{1}}-\nu_{\tilde{a}_{3}}\right| \leq\left|\nu_{\tilde{a}_{1}}-\nu_{\tilde{a}_{2}}\right|+\left|\nu_{\tilde{a}_{2}}-\nu_{\tilde{a}_{3}}\right|,
$$

so we have:

$$
\begin{aligned}
& \frac{\max \left(\left|u_{\tilde{a}_{1}}-u_{\tilde{a}_{3}}\right|,\left|\nu_{\tilde{a}_{1}}-\nu_{\tilde{a}_{3}}\right|\right)}{2} \\
& \leq \frac{\max \left(\left|u_{\tilde{a}_{1}}-u_{\tilde{a}_{2}}\right|,\left|\nu_{\tilde{a}_{1}}-\nu_{\tilde{a}_{2}}\right|\right)}{2} \\
& +\frac{\max \left(\left|u_{\tilde{a}_{2}}-u_{\tilde{a}_{3}}\right|,\left|\nu_{\tilde{a}_{1}}-\nu_{\tilde{a}_{3}}\right|\right)}{2} .
\end{aligned}
$$

By the same reason, we can get:

$$
\begin{aligned}
& \left|a_{1}-a_{3}\right| \leq\left|a_{1}-a_{2}\right|+\left|a_{2}-a_{3}\right|, \\
& \left|b_{1}-b_{3}\right| \leq\left|b_{1}-b_{2}\right|+\left|b_{2}-b_{3}\right|,
\end{aligned}
$$

and:

$$
\left|c_{1}-c_{3}\right| \leq\left|c_{1}-c_{2}\right|+\left|c_{2}-c_{3}\right| \text {. }
$$

Hence,

$$
d\left(\tilde{a}_{1}, \tilde{a}_{3}\right) \leq d\left(\tilde{a}_{1}, \tilde{a}_{2}\right)+d\left(\tilde{a}_{2}, \tilde{a}_{3}\right)
$$

That is to say, the proposed distance measure satisfies (iv) of Theorem 1.

For Example 1, by using Eq. (5), we have $d\left(\tilde{a}_{1}, \tilde{a}_{2}\right)=0.5$ and $d\left(\tilde{a}_{1}, \tilde{a}_{3}\right)=0.375$. Thus, $d\left(\tilde{a}_{1}, \tilde{a}_{2}\right)>d\left(\tilde{a}_{1}, \tilde{a}_{3}\right)$. It is expected that the distance between $\tilde{a}_{1}$ and $\tilde{a}_{2}$ is larger than the distance between $\tilde{a}_{1}$ and $\tilde{a}_{3}$, since they are ordered as $\tilde{a}_{1}>\tilde{a}_{3}>$ $\tilde{a}_{2}$ according to ranking method proposed by $\mathrm{Li}$ et al. [13,14]. Similarly, for Example 2, by using Eq. (5), we have $d\left(\tilde{a}_{4}, \tilde{a}_{5}\right)=0.1$, and $d\left(\tilde{a}_{4}, \tilde{a}_{6}\right)=0.2$. Thus, $d\left(\tilde{a}_{4}, \tilde{a}_{5}\right)<d\left(\tilde{a}_{4}, \tilde{a}_{6}\right)$, which is in agreement with the ranking method $[13,14]$. Therefore, the proposed distance measure is more reasonable. 


\subsection{Weighted average operator of TIFNs}

Definition 7 [26]. For a set of TIFNs $\tilde{a}_{i}(i=1$, $2, \cdots, n)$ that has associated importance weight vector $\mathbf{w}=\left\{w_{1}, w_{2}, \cdots, w_{n}\right\}^{T}$ with $w_{i} \in[0,1]$ and $\sum_{i=1}^{n} w_{i}=$ 1, we call:

$$
\begin{aligned}
& \operatorname{TIF-WA}\left(\tilde{a}_{1}, \tilde{a}_{2}, \cdots, \tilde{a}_{n}\right)=\sum_{i=1}^{n} w_{i} \tilde{a}_{i} \\
&=\left(\left(\sum_{i=1}^{n} w_{i} a_{i}, \sum_{i=1}^{n} w_{i} b_{i}, \sum_{i=1}^{n} w_{i} c_{i}\right) ;\right. \\
&\left.\min _{1 \leq i \leq n}\left\{u_{\tilde{a}_{i}}\right\}, \max _{1 \leq i \leq n}\left\{\nu_{\tilde{a}_{i}}\right\}\right),
\end{aligned}
$$

the Triangular Intuitionistic Fuzzy Weighted Average operator (TIF-WA).

\section{A new value index of TIFNs and ranking method}

\subsection{The mean value of TIFNs}

In this subsection, analogous to the idea of Nan et al. [20], the mean value index of a TIFN is defined as follows.

Definition 8. Let $\tilde{a}_{\alpha}$ and $\tilde{a}_{\beta}$ be a $\alpha$-cut set and a $\beta$-cut set of a TIFN $\tilde{a}=\left((a, b, c) ; u_{\tilde{a}}, \nu_{\tilde{a}}\right)$, respectively, and $m\left(\tilde{a}_{\alpha}\right)$ and $m\left(\tilde{a}_{\beta}\right)$ be the average values of $\tilde{a}_{\alpha}$ and $\tilde{a}_{\beta}$, i.e.:

$$
m\left(\tilde{a}_{\alpha}\right)=\frac{1}{2}\left[L_{\tilde{a}}(\alpha)+R_{\tilde{a}}(\alpha)\right]=\frac{2 \alpha b+\left(u_{\tilde{a}}-\alpha\right)(a+c)}{2 u_{\tilde{a}}},
$$

and:

$$
\begin{aligned}
m\left(\tilde{a}_{\beta}\right) & =\frac{1}{2}\left[L_{\tilde{a}}(\beta)+R_{\tilde{a}}(\beta)\right] \\
& =\frac{2(1-\beta) b+\left(\beta-\nu_{\tilde{a}}\right)(a+c)}{2\left(1-\nu_{\tilde{a}}\right)} .
\end{aligned}
$$

Then, the mean values of the membership and nonmembership functions are, respectively, defined as:

$$
M_{\mu}(\tilde{a})=\int_{0}^{u_{\bar{\alpha}}} m\left(\tilde{a}_{\alpha}\right) f(\alpha) d \alpha,
$$

and:

$$
M_{v}(\tilde{a})=\int_{\nu_{\bar{\alpha}}}^{1} m\left(\tilde{a}_{\beta}\right) g(\beta) d \beta,
$$

where function $f(\alpha)$ is non-negative and nondecreasing on the interval $\left[0, u_{\tilde{a}}\right]$ and satisfies the conditions: $f(0)=0$ and $\int_{0}^{u_{\bar{a}}} f(\alpha) d \alpha=\mu_{\tilde{a}}$; function $g(\beta)$ is non-negative and non-increasing on the interval $\left[\nu_{\tilde{a}}, 1\right]$ and satisfies the conditions: $g(1)=0$ and $\int_{\nu_{\bar{a}}}^{1} g(\beta) d \beta=1-\nu_{\tilde{a}}$.
Definition 9. For TIFN $\tilde{a}=\left((a, b, c) ; u_{\tilde{a}}, \nu_{\tilde{a}}\right)$, the mean-index is defined as:

$$
M(\tilde{a})=\frac{1}{2}\left[M_{\mu}(\tilde{a})+M_{v}(\tilde{a})\right] .
$$

Obviously, $M(\tilde{a})$ synthetically reflects the value of membership and non-membership functions.

Remark 1. If $f(\alpha)$ and $g(\beta)$ are, respectively, chosen as follows:

$$
f(\alpha)=\frac{2 \alpha}{u_{\tilde{a}}}\left(\alpha \in\left[0, u_{\tilde{a}}\right]\right),
$$

and:

$$
g(\beta)=\frac{2(1-\beta)}{1-\nu_{\tilde{a}}}\left(\beta \in\left[\nu_{\tilde{a}}, 1\right]\right) .
$$

According to Eqs. (7) and (12), the mean value of the membership function of TIFN $\tilde{a}$ is calculated as follows:

$$
\begin{aligned}
M_{\mu}(\tilde{a}) & =\int_{0}^{u_{\tilde{\alpha}}} \frac{2 \alpha b+\left(u_{\tilde{a}}-\alpha\right)(a+c)}{2 u_{\tilde{a}}} \frac{2 \alpha}{u_{\tilde{a}}} d \alpha \\
& =\frac{1}{6}(a+4 b+c) u_{\tilde{a}} .
\end{aligned}
$$

By Eqs. (8) and (13), the mean value of the nonmembership function of TIFN $\tilde{a}$ is calculated as follows:

$$
\begin{aligned}
M_{v}(\tilde{a}) & =\int_{\nu_{\bar{\alpha}}}^{1} \frac{2(1-\beta) b+\left(\beta-\nu_{\tilde{a}}\right)(a+c)}{2\left(1-\nu_{\tilde{a}}\right)} \frac{2(1-\beta)}{1-\nu_{\tilde{a}}} d \beta \\
& =\frac{1}{6}(a+4 b+c)\left(1-\nu_{\tilde{a}}\right) .
\end{aligned}
$$

Further, by Eqs. (11), (14), and (15), we have:

$$
M(\tilde{a})=\frac{1}{12}(a+4 b+c)\left(1-\nu_{\tilde{a}}+u_{\tilde{a}}\right) .
$$

According to the condition $0 \leq u_{\tilde{a}}+\nu_{\tilde{a}} \leq 1$, it is directly derived from Eqs. (14) and (15) that $\frac{1}{6}(a+$ $4 b+c) u_{\tilde{a}} \leq \frac{1}{6}(a+4 b+c)\left(1-\nu_{\tilde{a}}\right)$, i.e., $M_{\mu}(\tilde{a}) \leq M_{v}(\tilde{a})$. Thus, the mean values of the membership and nonmembership functions of TIFN $\tilde{a}=\left((a, b, c) ; u_{\tilde{a}}, \nu_{\tilde{a}}\right)$ can be expressed as interval-valued form $\tilde{M}(\tilde{a})=$ $\left[M_{\mu}(\tilde{a}), M_{v}(\tilde{a})\right]$.

\subsection{The variance value and standard deviation value of TIFNs}

Definition 10. The variance indices of the membership and non-membership functions for TIFN $\tilde{a}=$ $\left((a, b, c) ; u_{\tilde{a}}, \nu_{\tilde{a}}\right)$ are, respectively, defined as:

$$
V_{\mu}(\tilde{a})=\int_{0}^{u_{\bar{\alpha}}}\left[m\left(\tilde{a}_{\alpha}\right)-M_{\mu}(\tilde{a})\right]^{2} f(\alpha) d \alpha,
$$


and:

$$
V_{v}(\tilde{a})=\int_{v_{\bar{\alpha}}}^{1}\left[m\left(\tilde{a}_{\beta}\right)-M_{v}(\tilde{a})\right]^{2} g(\beta) d \beta .
$$

Definition 11. For TIFN $\tilde{a}=\left((a, b, c) ; u_{\tilde{a}}, \nu_{\tilde{a}}\right)$, the variance-index is defined as:

$$
V(\tilde{a})=\frac{1}{2}\left[V_{\mu}(\tilde{a})+V_{v}(\tilde{a})\right] .
$$

Obviously, $V(\tilde{a})$ synthetically reflects the variance value of membership and non-membership functions.

Remark 2. Weighting functions $f(\alpha)$ and $g(\beta)$ are chosen as Eqs. (12) and (13), respectively. According to Eqs. (7), (8), and (16)-(18), we have:

$$
V_{\mu}(\tilde{a})=\left[\frac{1}{36}(a+4 b+c)^{2}\left(1-u_{\tilde{a}}\right)^{2}+\frac{1}{72}(2 b-a-c)^{2}\right] u_{\tilde{a}}
$$

and:

$V_{v}(\tilde{a})=\left[\frac{1}{36}(a+4 b+c)^{2}\left(\nu_{\tilde{a}}\right)^{2}+\frac{1}{72}(2 b-a-c)^{2}\right]\left(1-\nu_{\tilde{a}}\right)$.

Further, by Eqs. (19)-(21), we have:

$$
\begin{aligned}
V(\tilde{a})= & \frac{1}{72}(a+4 b+c)^{2}\left[u_{\tilde{a}}\left(1-u_{\tilde{a}}\right)^{2}+\left(1-\nu_{\tilde{a}}\right)\left(\nu_{\tilde{a}}\right)^{2}\right] \\
& +\frac{1}{144}(2 b-a-c)^{2}\left(1+u_{\tilde{a}}-\nu_{\tilde{a}}\right) .
\end{aligned}
$$

Definition 12. The standard deviation indices of the membership and non-membership functions for TIFN $\tilde{a}=\left((a, b, c) ; u_{\tilde{a}}, \nu_{\tilde{a}}\right)$ are, respectively, defined as:

$$
\begin{aligned}
D_{\mu}(\tilde{a}) & =\sqrt{V_{\mu}(\tilde{a})} \\
& =\sqrt{\left[\frac{1}{36}(a+4 b+c)^{2}\left(1-u_{\tilde{a}}\right)^{2}+\frac{1}{72}(2 b-a-c)^{2}\right] u_{\tilde{a}}}
\end{aligned}
$$

and:

$$
\begin{aligned}
D_{v}(\tilde{a}) & =\sqrt{V_{v}(\tilde{a})} \\
& =\sqrt{\left[\frac{1}{36}(a+4 b+c)^{2}\left(\nu_{\tilde{a}}\right)^{2}+\frac{1}{72}(2 b-a-c)^{2}\right]\left(1-\nu_{\tilde{a}}\right) .}
\end{aligned}
$$

Definition 13. For TIFN $\tilde{a}=\left((a, b, c) ; u_{\tilde{a}}, \nu_{\tilde{a}}\right)$, the standard deviation index is defined as:

$$
D(\tilde{a})=\frac{1}{2}\left[D_{\mu}(\tilde{a})+D_{v}(\tilde{a})\right] .
$$

Similarly, $D(\tilde{a})$ synthetically reflects the standard deviation value of membership and non-membership functions.

\subsection{A novel ranking method of TIFNs}

In this subsection, a novel order relation of TIFNs is defined as follows.

Definition 14. Let $M(\tilde{a})$ and $D(\tilde{a})$ be the comprehensive mean-index and standard deviation index of TIFN $\tilde{a}=\left((a, b, c) ; u_{\tilde{a}}, \nu_{\tilde{a}}\right)$. The rank index for TIFN $\tilde{a}$ is defined as follows:

$$
R_{\lambda}(\tilde{a})=M(\tilde{a})-\lambda D(\tilde{a})
$$

where $\lambda \in[0,1]$ is a weight, which represents the DM's risk preference. $\lambda \in[0,1 / 2)$ shows that the DM prefers risk; $\lambda \in(1 / 2,1]$ shows that the DM prefers keeping conservative; $\lambda=1 / 2$ shows that the DM maintains neutrality. Therefore, the rank index may reflect the DM's subjective attitude to the TIFN.

Let $\tilde{a}_{1}=\left(\left(a_{1}, b_{1}, c_{1}\right) ; \mu_{\tilde{a}_{1}}, \nu_{\tilde{a}_{1}}\right)$ and $\tilde{a}_{2}=\left(\left(a_{2}, b_{2}\right.\right.$, $\left.\left.c_{2}\right) ; \mu_{\tilde{a}_{2}}, \nu_{\tilde{a}_{2}}\right)$ be two TIFNs. According to their comprehensive mean-index and standard deviation index, we propose a new lexicographic ranking method for TIFNs, which can be summarized as follows:

(i) If $R_{\lambda}\left(\tilde{a}_{1}\right)<R_{\lambda}\left(\tilde{a}_{2}\right)$ for the same given $\lambda$, then $\tilde{a}_{1}$ is smaller than $\tilde{a}_{2}$, denoted by $\tilde{a}_{1}<\tilde{a}_{2}$;

(ii) If $R_{\lambda}\left(\tilde{a}_{1}\right)>R_{\lambda}\left(\tilde{a}_{2}\right)$, then $\tilde{a}_{1}$ is bigger than $\tilde{a}_{2}$, denoted by $\tilde{a}_{1}>\tilde{a}_{2}$;

(iii) If $R_{\lambda}\left(\tilde{a}_{1}\right)=R_{\lambda}\left(\tilde{a}_{2}\right)$, then $\tilde{a}_{1}$ and $\tilde{a}_{2}$ represent the same information, denoted by $\tilde{a}_{1}=\tilde{a}_{2}$.

Example 3. Consider two TIFNs:

$$
\tilde{a}_{1}=((0.3,0.45,0.9) ; 0.9,0.1)
$$

and:

$$
\tilde{a}_{2}=((0.2,0.5,0.8) ; 0.9,0.1) .
$$

Intuitively, the ranking order is not equal. However, by method [14], we have $V_{\mu}\left(\tilde{a}_{1}\right)=V_{v}\left(\tilde{a}_{1}\right)=0.405$, $V_{\mu}\left(\tilde{a}_{2}\right)=V_{v}\left(\tilde{a}_{2}\right)=0.405, A_{\mu}\left(\tilde{a}_{1}\right)=A_{v}\left(\tilde{a}_{1}\right)=0.081$, $A_{\mu}\left(\tilde{a}_{2}\right)=A_{v}\left(\tilde{a}_{2}\right)=0.081$, and obviously, $V_{\lambda}\left(\tilde{a}_{1}\right)=$ $V_{\lambda}\left(\tilde{a}_{2}\right)$ and $A_{\lambda}\left(\tilde{a}_{1}\right)=A_{\lambda}\left(\tilde{a}_{2}\right)$; therefore, the ranking order is $\tilde{a}_{1}=\tilde{a}_{2}$. Namely, the ranking method [14] cannot distinguish these fuzzy numbers. According to Definition 1, the ranking order is $\tilde{a}_{1}<\tilde{a}_{2}$ intuitively. By the proposed method in this paper, we get $R_{\lambda}\left(\tilde{a}_{1}\right)=$ $0.45-0.058 \lambda, \quad R_{\lambda}\left(\tilde{a}_{2}\right)=0.45-0.047 \lambda$, obviously, $R_{\lambda}\left(\tilde{a}_{1}\right)<R_{\lambda}\left(\tilde{a}_{2}\right)$; thus, the ranking order is also $\tilde{a}_{1}<\tilde{a}_{2}$, which shows the validation of the proposed method in this paper.

\section{A new method for MAGDM with TIFNs using the mean-index and variance-index ranking procedures}

In this section, we propose a new method for solving MAGDM with unknown weight information under TIF environment. 


\subsection{The description of MAGDM problems with TIFNs}

MAGDM is the procedure to find the best alternative among a set of feasible alternatives that are characterized by usually multiple conflicting attributes. Let $D=\left\{D_{1}, D_{2}, \cdots, D_{p}\right\}$ be a group of DecisionMakers (DMs) that have associated importance weight vector $\mathbf{u}=\left\{u_{1}, u_{2}, \cdots, u_{p}\right\}^{T}$ with $u_{k} \in[0,1]$ and $\sum_{k=1}^{p} u_{k}=1$. Let $S=\left\{S_{1}, S_{2}, \cdots, S_{m}\right\}$ be a discrete set of alternatives, and $A=\left\{a_{1}, a_{2}, \cdots, a_{n}\right\}$ be a set of attributes. The attribute weight vector given by $\mathrm{DM} D_{k}$ is $\mathbf{w}^{k}=\left(w_{1}^{k}, w_{2}^{k}, \cdots, w_{n}^{k}\right)^{T}$, such that $w_{j}^{k} \in[0,1]$ and $\sum_{j=1}^{n} w_{j}^{k}=1$. Suppose that the preference value of alternative $S_{i}(i=1,2, \cdots, m)$ with respect to attribute $a_{j}(j=1,2, \cdots, n)$ is a TIFN $\tilde{x}_{i j}^{k}=\left(\left(a_{i j}^{k^{\prime}}, b_{i j}^{k^{\prime}}, c_{i j}^{k^{\prime}}\right) ; u_{\tilde{x}_{i j}^{k}}, \nu_{\tilde{x}_{i j}^{k}}\right)$, given by DM $D_{k}$ $(k=1,2, \cdots, p)$. Thus, the MAGDM problem with TIFNs can be expressed concisely in the matrix format as $\tilde{\mathbf{A}}^{k}=\left(\tilde{x}_{i j}^{k}\right)_{m \times n}(k=1,2, \cdots, p)$.

Since different physical dimensions may be measured in different ways, matrix $\tilde{\mathbf{A}}^{k}=\left(\tilde{x}_{i j}^{k}\right)_{m \times n}$ needs to be normalized into matrix $\tilde{\mathbf{R}}^{k}=\left(\tilde{r}_{i j}^{k}\right)_{m \times n}$, where $\tilde{r}_{i j}^{k}=\left(\left(a_{i j}^{k}, b_{i j}^{k}, c_{i j}^{k}\right) ; u_{\tilde{r}_{i j}^{k}}, \nu_{\tilde{r}_{i j}^{k}}\right)$. In this paper, in order to make the ranges of normalized TIFNs belong to $[0,1]$, we employ the following normalized transformation:

$$
\begin{gathered}
\tilde{r}_{i j}^{k}=\left(\left(\frac{a_{i j}^{k^{\prime}}}{c_{j}^{+}}, \frac{b_{i j}^{k^{\prime}}}{c_{j}^{+}}, \frac{c_{i j}^{k^{\prime}}}{c_{j}^{+}}\right) ; u_{\tilde{r}_{i j}^{k}}, \nu_{\tilde{r}_{i j}^{k}}\right) \\
(i=1,2, \cdots, m ; j \in B),
\end{gathered}
$$

and:

$$
\begin{gathered}
\tilde{r}_{i j}^{k}=\left(\left(\frac{a_{j}^{-}}{c_{i j}^{k^{\prime}}}, \frac{a_{j}^{-}}{b_{i j}^{k^{\prime}}}, \frac{a_{j}^{-}}{a_{i j}^{k^{\prime}}}\right) ; u_{\tilde{r}_{i j}^{k}}, \nu_{\tilde{r}_{i j}^{k}}\right), \\
(i=1,2, \cdots, m ; j \in C),
\end{gathered}
$$

where:

$$
\begin{aligned}
& c_{j}^{+}=\max \left\{c_{i j}^{k^{\prime}} \mid i=1,2, \cdots, m\right\} \\
& a_{j}^{-}=\min \left\{a_{i j}^{k^{\prime}} \mid i=1,2, \cdots, m\right\} \\
& u_{\tilde{r}_{i j}^{k}}=u_{\tilde{x}_{i j}^{k}}, \quad v_{\tilde{r}_{i j}^{k}}=\nu_{\tilde{x}_{i j}^{k}} .
\end{aligned}
$$

$B$ and $C$ are the subscript sets of benefit and cost attribute sets, respectively.

\subsection{The approach to determining attributes weights}

Entropy is based on the concept of probability and measures the discrimination power of attributes when applied to MADM [30]. The bigger the amount of information is in each attribute, the less the entropy is, and vice versa. In other words, if the entropy measure for a given attribute is low, this attribute will be given to a higher weight, and vice versa. To deal with attribute value with TIFNs, fuzzy decision matrix, $\tilde{\mathbf{R}}^{k}=\left(\tilde{r}_{i j}^{k}\right)_{m \times n}$, should be transformed into a crisp matrix. According to normalized decision matrix, $\tilde{\mathbf{R}}^{k}=\left(\tilde{r}_{i j}^{k}\right)_{m \times n}$, we identify that $\gamma^{+}=<(1,1,1) ; 1,0>$ and $\gamma^{-}=<(0,0,0) ; 0,1>$ are the largest TIFNs and the smallest TIFNs. The reasons are:

1. Since $\tilde{\mathbf{R}}^{k}=\left(\tilde{r}_{i j}^{k}\right)_{m \times n}$ is a normalized decision matrix, it is easy to define $\gamma^{+}$as the largest TIFN and $\gamma^{-}$as the smallest TIFN;

2. $\gamma^{+}$and $\gamma^{-}$can be regarded as a unified bound for all individual decision matrices.

By using Eq. (5), the separation measures of $\tilde{r}_{i j}^{k}$ from the largest TIFNs are given as:

$$
\begin{aligned}
d\left(\tilde{r}_{i j}^{k}, \gamma^{+}\right)= & \frac{1}{6}\left(\left|a_{i j}^{k}-1\right|+\left|b_{i j}^{k}-1\right|+\left|c_{i j}^{k}-1\right|\right) \\
& +\frac{1}{8}\left(\left|u_{\tilde{r}_{i j}^{k}}-1\right|+\left|\nu_{\tilde{r}_{i j}^{k}}-0\right|\right) \\
& +\frac{1}{4} \max \left(\left|u_{\tilde{r}_{i j}^{k}}-1\right|,\left|\nu_{\tilde{r}_{i j}^{k}}-0\right|\right) .
\end{aligned}
$$

Similarly, the separation measures of $\tilde{r}_{i j}^{k}$ value from the smallest TIFNs are given as:

$$
\begin{aligned}
d\left(\tilde{r}_{i j}^{k}, \gamma^{-}\right)= & \frac{1}{6}\left(a_{i j}^{k}+b_{i j}^{k}+c_{i j}^{k}\right)+\frac{1}{8}\left(u_{\tilde{r}_{i j}^{k}}+\left|\nu_{\tilde{r}_{i j}^{k}}-1\right|\right) \\
& +\frac{1}{4} \max \left(u_{\tilde{r}_{i j}^{k}},\left|\nu_{\tilde{r}_{i j}^{k}}-1\right|\right) .
\end{aligned}
$$

The relative closeness coefficient of TIFN $\tilde{r}_{i j}^{k}$ is defined as:

$$
h_{i j}^{k}=\frac{d\left(\tilde{r}_{i j}^{k}, \gamma^{-}\right)}{d\left(\tilde{r}_{i j}^{k}, \gamma^{-}\right)+d\left(\tilde{r}_{i j}^{k}, \gamma^{+}\right)} .
$$

Hence, according to Eq. (31), normalized decision matrix, $\tilde{\mathbf{R}}^{k}=\left(\tilde{r}_{i j}^{k}\right)_{m \times n}$, can be transformed into crisp relative closeness coefficient matrix, $\mathbf{H}^{k}=\left(h_{i j}^{k}\right)_{m \times n}$.

For matrix $\mathbf{H}^{k}$, the entropy measure of attribute $a_{j}$ for DM $D_{k}$ can be defined as:

$$
e_{j}^{k}=-\frac{1}{\ln (m)} \sum_{i=1}^{m}\left[h_{i j}^{k} \ln \left(h_{i j}^{k}\right)\right] \quad(j=1,2, \cdots, n) .
$$

Therefore, entropy weight $w_{j}$ of attribute $a_{j}$ for DM $D_{k}$ can be obtained as follows:

$$
w_{j}^{k}=-\frac{1-e_{j}^{k}}{\sum_{j=1}^{n} 1-e_{j}^{k}} \quad(j=1,2, \cdots, n) .
$$

From Eq. (33), attribute weight vector $\mathbf{w}^{k}=\left(w_{1}^{k}, w_{2}^{k}\right.$, $\left.\cdots, w_{n}^{k}\right)^{T}$ can be obtained for DM $D_{k}$. 


\subsection{Determining the weights of DMs}

In this section, we will elaborate on determining DM weights from another point of view. Inspired by the literatures $[29,31]$, based on the mean, the best decision should be the average of all individual decisions. The average decision is defined as:

$$
\tilde{\mathbf{R}}^{*}=\left(\tilde{r}_{i j}^{*}\right)_{m \times n}
$$

where $\tilde{r}_{i j}^{*}=\frac{1}{p} \sum_{k=1}^{p} \tilde{r}_{i j}^{k}(i \in m, j \in n)$. By comparing the individual decision with the average decision, we can give the definition of similarity of the individual decision with respect to the average decisions.

Definition 15. In a given MAGDM with TIFNs, the similarity of the individual decision given by DM $D_{k}$ with respect to the average decision is defined as:

$$
S_{k}=\frac{1}{m n} \sum_{i=1}^{m} \sum_{j=1}^{n}\left(1-d\left(\tilde{r}_{i j}^{*}, \tilde{r}_{i j}^{k}\right)\right)
$$

where $d\left(\tilde{r}_{i j}^{*}, \tilde{r}_{i j}^{k}\right)$ is the distance between $\tilde{r}_{i j}^{*}$ and $\tilde{r}_{i j}^{k}$ defined in Definition 6. Clearly, $0 \leq S_{k} \leq 1$.

Generally, a DM's opinion is more important if his/hers opinion is more similar to group's opinion. The larger the value of $S_{k}$ is, the more important the opinion of DM $D_{k}$ is. Thus, $S_{k}$ can measure the importance of DM $D_{k}$. After normalization, weight $u_{k}$ of DM $D_{k}$ can be obtained by:

$$
u_{k}=\frac{S_{k}}{\sum_{k=1}^{p} S_{k}} \quad(k=1,2, \cdots, p) .
$$

\subsection{The method for MAGDM with TIFNs}

Below, we develop a new method for solving the MAGDM problems with unknown attribute weights and DM weights under TIF environment, which can be summarized in detail as follows:

- Step 1. Form a group of DMs $D=\left\{D_{1}, D_{2}, \cdots\right.$, $\left.D_{p}\right\}$ and identify the set of alternatives $S=\left\{S_{1}, S_{2}\right.$, $\left.\cdots, S_{m}\right\}$ and the set of attributes $A=\left\{a_{1}, a_{2}, \cdots\right.$, $\left.a_{n}\right\}$;

- Step 2. DM $D_{k}$ gives his decision matrix, $\tilde{\mathbf{A}}^{k}=$ $\left(\tilde{x}_{i j}^{k}\right)_{m \times n}$;

- Step 3. Normalize TIFN decision matrix, $\tilde{\mathbf{A}}^{k}=$ $\left(\tilde{x}_{i j}^{k}\right)_{m \times n}$, into matrix $\tilde{\mathbf{R}}^{k}=\left(\tilde{r}_{i j}^{k}\right)_{m \times n}$ by Eqs. (27) and $(28)$;

- Step 4. Determine the weight vector of attributes $\mathbf{w}^{k}=\left(w_{1}^{k}, w_{2}^{k}, \cdots, w_{n}^{k}\right)^{T}$ for DM $D_{k}$ using the entropy method developed in Section 4.3;

- Step 5. Compute the individual overall attribute value of alternative $S_{i}$ for DM $D_{k}$ by Eq. (6) as follows:

$$
\begin{gathered}
\tilde{E}_{i}^{k}=\operatorname{TIF-WA}\left(\tilde{a}_{i 1}^{k}, \tilde{a}_{i 2}^{k}, \cdots, \tilde{a}_{i n}^{k}\right) \\
=\left(\left(\sum_{j=1}^{n} w_{j}^{k} a_{i j}^{k}, \sum_{j=1}^{n} w_{j}^{k} b_{i j}^{k}, \sum_{j=1}^{n} w_{j}^{k} c_{i j}^{k}\right) ;\right. \\
\left.\min _{1 \leq j \leq n}\left\{u_{\tilde{a}_{i j}}\right\}, \max _{1 \leq j \leq n}\left\{\nu_{\tilde{a}_{i j}}\right\}\right) .
\end{gathered}
$$

- Step 6. Calculate the weight vector of DMs $\mathbf{u}=$ $\left\{u_{1}, u_{2}, \cdots, u_{p}\right\}^{T}$ using Eqs. (34)-(36);

- Step 7. Utilize the TIF-WA operator:

$$
\tilde{E}_{i}=\operatorname{TIF}-W A\left(\tilde{E}_{i}^{1}, \tilde{E}_{i}^{2}, \cdots, \tilde{E}_{i}^{p}\right)=\sum_{k=1}^{p} u_{k} \tilde{E}_{i}^{k}
$$

to aggregate all $\tilde{E}_{i}^{k}(k=1,2, \cdots, p)$ into a collective value for alternative $S_{i}$;

- Step 8. Calculate mean-index, $M\left(\tilde{E}_{i}\right)$, and standard deviation index, $D\left(\tilde{E}_{i}\right)$, of each overall attribute value, $\tilde{E}_{i}$, for alternative $S_{i}$ by Eqs. (16) and (25);

- Step 9. Choose DM's preference information, $\lambda$, and compute ranking index, $R_{\lambda}\left(\tilde{E}_{i}\right)$, of each alternative by using Eq. (26);

- Step 10. Rank the alternatives according to ranking indices, $R_{\lambda}\left(\tilde{E}_{i}\right)$, and select the best one.

\section{A trustworthy service selection example and comparative analyses}

\subsection{Trustworthy service selection example}

Trustworthy service selection recommends the most trustworthy service suppliers based on the objective trust indictors. Online supplier selection is a common problem for many customers. When buying a desired item from an online supplier, it is an important precondition to assess the trustworthiness of the supplier. Trust has been identified as a fundamental factor in human relationships enabling cooperation to take place. Trust is an efficient mechanism to foster the cooperation between suppliers and customers, especially for online service selection based on electronic market, and to avoid potential transaction risks. The proposed method in this paper provides an effective framework for dealing with the selection of one supplier from a list of interested providers. In the following, a trustworthy supplier selection example will illustrate application of the proposed method.

A consumer desires to select a trustworthy seller to buy clothes. After preliminary screening, three candidate sellers, Handuyishe $\left(S_{1}\right)$, Niman $\left(S_{2}\right)$, and Liebo $\left(S_{3}\right)$, which are Taobao's clothing brand, remain for further evaluation. Based on the detailed seller ratings, the decision-making committee assesses the 
Table 1. The TIFN decision matrix given by $D_{1}$.

\begin{tabular}{cccccc}
\hline & $\boldsymbol{a}_{\mathbf{1}}$ & $\boldsymbol{a}_{\mathbf{2}}$ & $\boldsymbol{a}_{\mathbf{3}}$ & $\boldsymbol{a}_{\mathbf{4}}$ & $\boldsymbol{a}_{\mathbf{5}}$ \\
\hline $\boldsymbol{S}_{\mathbf{1}}$ & $((3.1,4.2,9.2) ; 0.6,0.1)$ & $((3.3,5.1,7.0) ; 0.5,0.4)$ & $((5.2,7.3,8.4) ; 0.5,0.2)$ & $((6.1,8.0,9.1) ; 0.7,0.3)$ & $((4.6,5.4,6.3) ; 0.5,0.3)$ \\
$\boldsymbol{S}_{\mathbf{2}}$ & $((4.2,5.2,10) ; 0.5,0.2)$ & $((5.3,6.1,7.3) ; 0.4 .0 .2)$ & $((4.3,5.3,6.2) ; 0.5,0.1)$ & $((6.0,7.2,8.1) ; 0.6,0.2)$ & $((3.1,4.5,10) ; 0.6,0.3)$ \\
$\boldsymbol{S}_{\mathbf{3}}$ & $((2.3,4.1,5.3) ; 0.5,0.3)$ & $((6.1,8.2,10) ; 0.7,0.1)$ & $((4.1,6.2,8.3) ; 0.5,0.1)$ & $((4.2,6.5,10) ; 0.5,0.5)$ & $((4.4,5.1,6.2) ; 0.6,0.2)$ \\
\hline
\end{tabular}

Table 2. The TIFN decision matrix given by $D_{2}$.

\begin{tabular}{cccccc}
\hline & $\boldsymbol{a}_{\mathbf{1}}$ & $\boldsymbol{a}_{\mathbf{2}}$ & $\boldsymbol{a}_{\mathbf{3}}$ & $\boldsymbol{a}_{\mathbf{4}}$ & $\boldsymbol{a}_{\mathbf{5}}$ \\
\hline $\boldsymbol{S}_{\mathbf{1}}$ & $((5.0,6.0,10) ; 0.3,0.1)$ & $((4.0,5.5,7.2) ; 0.6,0.4)$ & $((6.5,7.1,8.0) ; 0.5,0.2)$ & $((4.5,7.3,8.2) ; 0.7,0.3)$ & $((4.0,5.7,6.8) ; 0.4,0.2)$ \\
$\boldsymbol{S}_{\boldsymbol{2}}$ & $((3.4,4.5,7.8) ; 0.5,0.2)$ & $((4.5,5.6,6.7) ; 0.5 .0 .3)$ & $((3.4,4.5,10) ; 0.3,0.1)$ & $((4.6,5.7,7.8) ; 0.4,0.4)$ & $((5.3,6.4,10) ; 0.7,0.1)$ \\
$\boldsymbol{S}_{\boldsymbol{3}}$ & $((4.2,5.4,7.5) ; 0.5,0.2)$ & $((5.6,6.8,10) ; 0.7,0.1)$ & $((4.4,5.6,6.8) ; 0.6,0.2)$ & $((5.4,6.6,10) ; 0.4,0.3)$ & $((3.4,6.5,8.6) ; 0.5,0.3)$ \\
\hline
\end{tabular}

Table 3. The TIFN decision matrix given by $D_{3}$.

\begin{tabular}{cccccc}
\hline & $\boldsymbol{a}_{\mathbf{1}}$ & $\boldsymbol{a}_{\mathbf{2}}$ & $\boldsymbol{a}_{\mathbf{3}}$ & $\boldsymbol{a}_{\mathbf{4}}$ & $\boldsymbol{a}_{\mathbf{5}}$ \\
\hline $\boldsymbol{S}_{\mathbf{1}}$ & $((5.3,6.4,7.9) ; 0.4,0.2)$ & $((4.3,5.5,6.7) ; 0.6,0.2)$ & $((3.5,6.7,7.8) ; 0.3,0.4)$ & $((2.6,5.8,6.9) ; 0.5,0.1)$ & $((4.4,5.0,6.0) ; 0.3,0.3)$ \\
$\boldsymbol{S}_{\mathbf{2}}$ & $((3.4,5.0,10) ; 0.4,0.3)$ & $((4.5,6.6,7.0) ; 0.5 .0 .1)$ & $((2.4,5.0,6.6) ; 0.4,0.4)$ & $((3.6,5.7,8.0) ; 0.6,0.3)$ & $((4.3,7.4,10) ; 0.3,0.4)$ \\
$\boldsymbol{S}_{\mathbf{3}}$ & $((5.2,6.4,8.5) ; 0.7,0.1)$ & $((3.6,5.8,10) ; 0.6,0.4)$ & $((5.4,6.0,10) ; 0.4,0.3)$ & $((2.4,8.6,10) ; 0.6,0.3)$ & $((4.4,5.0,7.6) ; 0.5,0.1)$ \\
\hline
\end{tabular}

Table 4. The TIFN decision matrix given by $D_{4}$.

\begin{tabular}{cccccc}
\hline & $\boldsymbol{a}_{\mathbf{1}}$ & $\boldsymbol{a}_{\mathbf{2}}$ & $\boldsymbol{a}_{\mathbf{3}}$ & $\boldsymbol{a}_{\mathbf{4}}$ & $\boldsymbol{a}_{\mathbf{5}}$ \\
\hline $\boldsymbol{S}_{\mathbf{1}}$ & $((5.3,6.4,7.9) ; 0.4,0.4)$ & $((3.7,5.2,7.1) ; 0.5,0.2)$ & $((2.5,4.7,6.6) ; 0.3,0.4)$ & $((3.8,4.8,7.9) ; 0.7,0.3)$ & $((4.9,5.8,6.3) ; 0.3,0.4)$ \\
$\boldsymbol{S}_{\mathbf{2}}$ & $((5.4,8.5,10) ; 0.4,0.2)$ & $((6.4,7.6,8.8) ; 0.4 .0 .4)$ & $((5.4,6.5,10) ; 0.6,0.4)$ & $((3.4,5.7,7.9) ; 0.7,0.3)$ & $((5.3,6.7,10) ; 0.5,0.4)$ \\
$\boldsymbol{S}_{\mathbf{3}}$ & $((2.7,4.8,5.9) ; 0.6,0.2)$ & $((6.3,8.0,10) ; 0.6,0.2)$ & $((3.4,4.6,6.8) ; 0.7,0.2)$ & $((5.4,7.6,10) ; 0.4,0.3)$ & $((3.1,6.4,7.7) ; 0.5,0.1)$ \\
\hline
\end{tabular}

four candidate sellers according to five trust factors: product quality $\left(a_{1}\right)$, service attitude $\left(a_{2}\right)$, technology security $\left(a_{3}\right)$, website design embodies appearance and ease use $\left(a_{4}\right)$, and shipping speed $\left(a_{5}\right)$. Four DMs adopt the ten-mark system to evaluate the three candidate sellers based on five attributes. Using statistical methods, the ratings of the candidate sellers with respect to the attributes can be represented as TIFNs, shown in Tables 1-4.

- Step 1 and Step 2. Four DMs evaluate the candidate sellers with TIFNs, and the decision matrixes $\tilde{A}^{k}=\left(\tilde{x}_{i j}^{k}\right)_{m \times n}(k=1,2, \cdots, p)$ of DMs are listed in Tables 1-4;

- Step 3. By Using Eqs. (27) and (28), normalized TIFN decision matrixes, $\tilde{R}^{k}=\left(\tilde{r}_{i j}^{k}\right)_{m \times n}(k=$ $1,2, \cdots, p)$, of DMs are obtained and listed in Tables 5-8;

- Step 4. Determine the largest TIFNs and the smallest TIFNs in decision matrix $\tilde{R}^{k}$ as follows:

$$
\gamma^{+}=<(1,1,1) ; 1,0>, \quad \gamma^{-}=<(0,0,0) ; 0,1>.
$$

By using Eqs. (29)-(31), relative closeness coefficient matrixes, $\mathbf{H}^{k}=\left(h_{i j}^{k}\right)_{m \times n}$, of DMs are given as in Table 9. Employed Eqs. (32) and (33), the attribute weight vectors for each DMs can be calculated as follows:

$$
\begin{aligned}
& \mathbf{w}^{1}=(0.2324,0.5330,0.0476,0.1354,0.1516)^{T}, \\
& \mathbf{w}^{2}=(0.0254,0.3971,0.0712,0.1783,0.3280)^{T}, \\
& \mathbf{w}^{3}=(0.3011,0.0276,0.3603,0.0851,0.2258)^{T}, \\
& \mathbf{w}^{4}=(0.1413,0.1649,0.4866,0.0042,0.2031)^{T} .
\end{aligned}
$$

- Step 5. According to Eq. (37), we can utilize the TIF-WA operator to derive the attribute values $\left(\tilde{a}_{i 1}^{k}, \tilde{a}_{i 2}^{k}, \cdots, \tilde{a}_{i n}^{k}\right)$ into overall attribute value $\tilde{E}_{i}^{k}$, listed in Table 10;

- Step 6. Using Eq. (34), the average of all individual decisions is obtained and listed in Table 11. Then, the weight vector of DMs can be calculated using Eqs. (35) and (36) as:

$$
\mathbf{u}=\{0.2447,0.2492,0.2523,0.2538\}^{T} .
$$

- Step 7. According to Eq. (38), we can utilize the TIF-WA operator to aggregate all $\tilde{E}_{i}^{k}(k=$ $1,2, \cdots, p)$ of each DM into collective value $\tilde{E}_{i}$ for each alternative as follows:

$$
\tilde{E}_{1}=((0.396,0.570,0.735) ; 0.3,0.4)
$$


Table 5. The normalized TIFN decision matrix given by $D_{1}$.

\begin{tabular}{cccccc}
\hline & $\boldsymbol{a}_{\mathbf{1}}$ & $\boldsymbol{a}_{\mathbf{2}}$ & $\boldsymbol{a}_{\mathbf{3}}$ & $\boldsymbol{a}_{\mathbf{4}}$ & $\boldsymbol{a}_{\mathbf{5}}$ \\
\hline \multirow{2}{*}{$\boldsymbol{S}_{\mathbf{1}}$} & $((0.31,0.42,0.92) ;$ & $((0.33,0.51,0.7) ;$ & $((0.52,0.73,0.84) ;$ & $((0.61,0.8,0.91) ;$ & $((0.46,0.54,0.63) ;$ \\
& $0.6,0.1)$ & $0.5,0.4)$ & $0.5,0.2)$ & $0.7,0.3)$ & $0.5,0.3)$ \\
$\boldsymbol{S}_{\mathbf{2}}$ & $((0.42,0.52,1) ;$ & $((0.53,0.61,0.73) ;$ & $((0.43,0.53,0.62) ;$ & $((0.6,0.72,0.81) ;$ & $((0.31,0.45,1) ;$ \\
& $0.5,0.2)$ & $0.4 .0 .2)$ & $0.5,0.1)$ & $0.6,0.2)$ & $0.6,0.3)$ \\
$\boldsymbol{S}_{\mathbf{3}}$ & $((0.23,0.41,0.53) ;$ & $((0.61,0.82,1) ;$ & $((0.41,0.62,0.83) ;$ & $((0.42,0.65,1) ;$ & $((0.44,0.51,0.62) ;$ \\
& $0.5,0)$. & $0.7,0.1)$ & $0.5,0.1)$ & $0.5,0.5)$ & $0.6,0.2)$ \\
\hline
\end{tabular}

Table 6. The normalized TIFN decision matrix given by $D_{2}$.

\begin{tabular}{cccccc}
\hline & $\boldsymbol{a}_{\mathbf{1}}$ & $\boldsymbol{a}_{\mathbf{2}}$ & $\boldsymbol{a}_{\mathbf{3}}$ & $\boldsymbol{a}_{\mathbf{4}}$ & $\boldsymbol{a}_{\mathbf{5}}$ \\
\hline \multirow{2}{*}{$\boldsymbol{S}_{\mathbf{1}}$} & $((0.5,0.6,1) ;$ & $((0.4,0.55,0.72) ;$ & $((0.65,0.71,0.8) ;$ & $((0.45,0.73,0.82) ;$ & $((0.4,0.57,0.68) ;$ \\
& $0.3,0.1)$ & $0.6,0.4)$ & $0.5,0.2)$ & $0.7,0.3)$ & $0.4,0.2)$ \\
$\boldsymbol{S}_{\mathbf{2}}$ & $((0.34,0.45,0.78) ;$ & $((0.45,0.56,0.67) ;$ & $((0.34,0.45,1) ;$ & $((0.46,0.57,0.78) ;$ & $((0.53,0.64,1) ;$ \\
& $0.5,0.2)$ & $0.5 .0 .3)$ & $0.3,0.1)$ & $0.4,0.4)$ & $0.7,0.1)$ \\
$\boldsymbol{S}_{\mathbf{3}}$ & $((0.42,0.54,0.75) ;$ & $((0.56,0.68,1) ;$ & $((0.44,0.56,0.68) ;$ & $((0.54,0.66,1) ;$ & $((0.34,0.65,0.86) ;$ \\
& $0.5,0.2)$ & $0.7,0.1)$ & $0.6,0.2)$ & $0.4,0.3)$ & $0.5,0.3)$ \\
\hline
\end{tabular}

Table 7. The normalized TIFN decision matrix given by $D_{3}$.

\begin{tabular}{cccccc}
\hline & $\boldsymbol{a}_{\mathbf{1}}$ & $\boldsymbol{a}_{\mathbf{2}}$ & $\boldsymbol{a}_{\mathbf{3}}$ & $\boldsymbol{a}_{\mathbf{4}}$ & $\boldsymbol{a}_{\mathbf{5}}$ \\
\hline \multirow{2}{*}{$\boldsymbol{S}_{\mathbf{1}}$} & $((0.53,0.64,0.79) ;$ & $((0.43,0.55,0.67) ;$ & $((0.35,0.67,0.78) ;$ & $((0.26,0.58,0.69) ;$ & $((0.44,0.5,0.6) ;$ \\
& $0.4,0.2)$ & $0.6,0.2)$ & $0.3,0.4)$ & $0.5,0.1)$ & $0.3,0.3)$ \\
$\boldsymbol{S}_{\mathbf{2}}$ & $((0.34,0.5,1) ;$ & $((0.45,0.66,0.7) ;$ & $((0.24,0.5,0.66) ;$ & $((0.36,0.57,0.8) ;$ & $((0.43,0.74,1) ;$ \\
& $0.4,0.3)$ & $0.5 .0 .1)$ & $0.4,0.4)$ & $0.6,0.3)$ & $0.3,0.4)$ \\
$\boldsymbol{S}_{\mathbf{3}}$ & $((0.52,0.64,0.85) ;$ & $((0.36,0.58,1) ;$ & $((0.54,0.6,1) ;$ & $((0.24,0.86,1) ;$ & $((0.44,0.5,0.76) ;$ \\
& $0.7,0)$ & $0.6,0.4)$ & $0.4,0.3)$ & $0.6,0.3)$ & $0.5,0.1)$ \\
\hline
\end{tabular}

Table 8. The normalized TIFN decision matrix given by $D_{4}$.

\begin{tabular}{cccccc}
\hline & $\boldsymbol{a}_{\mathbf{1}}$ & $\boldsymbol{a}_{\mathbf{2}}$ & $\boldsymbol{a}_{\mathbf{3}}$ & $\boldsymbol{a}_{\mathbf{4}}$ & $\boldsymbol{a}_{\mathbf{5}}$ \\
\hline \multirow{2}{*}{$\boldsymbol{S}_{\mathbf{1}}$} & $((0.53,0.64,0.79) ;$ & $((0.37,0.52,0.71) ;$ & $((0.25,0.47,0.66) ;$ & $((0.38,0.48,0.79) ;$ & $((0.49,0.58,0.63) ;$ \\
& $0.4,0.4)$ & $0.5,0.2)$ & $0.3,0.4)$ & $0.7,0.3)$ & $0.3,0.4)$ \\
$\boldsymbol{S}_{\mathbf{2}}$ & $((0.54,0.85,1) ;$ & $((0.64,0.76,0.88) ;$ & $((0.54,0.65,1) ;$ & $((0.34,0.57,0.79) ;$ & $((0.53,0.67,1) ;$ \\
& $0.4,0.2)$ & $0.4 .0 .4)$ & $0.6,0.4)$ & $0.7,0.3)$ & $0.5,0.4)$ \\
$\boldsymbol{S}_{\mathbf{3}}$ & $((0.27,0.48,0.59) ;$ & $((0.63,0.8,1) ;$ & $((0.34,0.46,0.68) ;$ & $((0.54,0.76,1) ;$ & $((0.31,0.64,0.77) ;$ \\
& $0.6,0.2)$ & $0.6,0.2)$ & $0.7,0.2)$ & $0.4,0.3)$ & $0.5,0.1)$ \\
\hline
\end{tabular}

Table 9. The relative closeness coefficient matrices of four DMs.

\begin{tabular}{|c|c|c|c|c|c|c|c|c|c|c|}
\hline & \multicolumn{5}{|c|}{$D_{1}$} & \multicolumn{5}{|c|}{$D_{2}$} \\
\hline & $a_{1}$ & $a_{2}$ & $a_{3}$ & $a_{4}$ & $a_{5}$ & $a_{1}$ & $a_{2}$ & $a_{3}$ & $a_{4}$ & $a_{5}$ \\
\hline$S_{1}$ & 0.64 & 0.53 & 0.69 & 0.74 & 0.54 & 0.63 & 0.58 & 0.67 & 0.68 & 0.57 \\
\hline$S_{2}$ & 0.64 & 0.58 & 0.60 & 0.70 & 0.62 & 0.58 & 0.58 & 0.59 & 0.55 & 0.75 \\
\hline \multirow[t]{3}{*}{$S_{3}$} & 0.49 & 0.79 & 0.65 & 0.60 & 0.61 & 0.60 & 0.76 & 0.62 & 0.63 & 0.60 \\
\hline & \multicolumn{5}{|c|}{$D_{3}$} & \multicolumn{5}{|c|}{$D_{4}$} \\
\hline & $a_{1}$ & $a_{2}$ & $a_{3}$ & $a_{4}$ & $a_{5}$ & $a_{1}$ & $a_{2}$ & $a_{3}$ & $a_{4}$ & $a_{5}$ \\
\hline$S_{1}$ & 0.62 & 0.62 & 0.52 & 0.60 & 0.51 & 0.57 & 0.58 & 0.46 & 0.63 & 0.51 \\
\hline$S_{2}$ & 0.58 & 0.64 & 0.48 & 0.61 & 0.58 & 0.68 & 0.60 & 0.67 & 0.63 & 0.64 \\
\hline$S_{3}$ & 0.72 & 0.67 & 0.62 & 0.67 & 0.62 & 0.57 & 0.72 & 0.62 & 0.65 & 0.62 \\
\hline
\end{tabular}


Table 10. Overall attribute value $\tilde{E}_{i}^{k}$.

\begin{tabular}{ccccc}
\hline & $\mathbf{D M}_{\mathbf{1}}$ & $\mathbf{D M}_{\mathbf{2}}$ & $\mathbf{D M}_{\mathbf{3}}$ & $\mathbf{D M}_{\mathbf{4}}$ \\
\hline $\boldsymbol{S}_{\mathbf{1}}$ & $((0.38,0.54,0.79) ; 0.4,0.4)$ & $((0.43,0.60,0.74) ; 0.3,0.4)$ & $((0.41,0.62,0.74) ; 0.3,0.4)$ & $((0.36,0.52,0.68) ; 0.3,0.4)$ \\
$\boldsymbol{S}_{\mathbf{2}}$ & $((0.50,0.59,0.81) ; 0.3,0.3)$ & $((0.47,0.58,0.82) ; 0.3 .0 .4)$ & $((0.32,0.56,0.83) ; 0.3,0.4)$ & $((0.55,0.70,0.98) ; 0.4,0.4)$ \\
$\boldsymbol{S}_{\mathbf{3}}$ & $((0.48,0.68,0.86) ; 0.5,0.5)$ & $((0.47,0.65,0.92) ; 0.4,0.3)$ & $((0.49,0.61,0.91) ; 0.4,0.3)$ & $((0.37,0.56,0.74) ; 0.4,0.3)$ \\
\hline
\end{tabular}

Table 11. The average of all individual decisions.

\begin{tabular}{cccccc}
\hline & $\boldsymbol{a}_{\mathbf{1}}$ & $\boldsymbol{a}_{\mathbf{2}}$ & $\boldsymbol{a}_{\mathbf{3}}$ & $\boldsymbol{a}_{\mathbf{4}}$ & $\boldsymbol{a}_{\mathbf{5}}$ \\
\hline \multirow{2}{*}{$\boldsymbol{S}_{\mathbf{1}}$} & $((0.47,0.57,0.87) ;$ & $((0.38,0.53,0.70) ;$ & $((0.44,0.65,0.81) ;$ & $((0.42,0.65,0.80) ;$ & $((0.45,0.55,0.64) ;$ \\
& $0.3,0.4)$ & $0.5,0.4)$ & $0.3,0.4)$ & $0.5,0.3)$ & $0.3,0.4)$ \\
$\boldsymbol{S}_{\mathbf{2}}$ & $((0.41,0.58,0.95) ;$ & $((0.52,0.65,0.83) ;$ & $((0.39,0.53,0.71) ;$ & $((0.44,0.61,0.8) ;$ & $((0.45,0.63,1) ;$ \\
& $0.4,0.3)$ & $0.3 .0 .4)$ & $0.3,0.4)$ & $0.4,0.4)$ & $0.3,0.4)$ \\
$\boldsymbol{S}_{\mathbf{3}}$ & $((0.36,0.52,0.68) ;$ & $((0.54,0.72,1) ;$ & $((0.43,0.56,0.80) ;$ & $((0.44,0.73,1) ;$ & $((0.38,0.58,0.75) ;$ \\
& $0.5,0.3)$ & $0.6,0.3)$ & $0.4,0.3)$ & $0.4,0.5)$ & $0.5,0.3)$ \\
\hline
\end{tabular}

Table 12. The ranking order with different DM's risk preference.

\begin{tabular}{cccccc}
\hline $\boldsymbol{\lambda}$ & $\boldsymbol{R}_{\boldsymbol{\lambda}}\left(\boldsymbol{S}_{\mathbf{1}}\right)$ & $\boldsymbol{R}_{\boldsymbol{\lambda}}\left(\boldsymbol{S}_{\mathbf{2}}\right)$ & $\boldsymbol{R}_{\boldsymbol{\lambda}}\left(\boldsymbol{S}_{\mathbf{3}}\right)$ & $\begin{array}{c}\text { Ranking } \\
\text { orders }\end{array}$ & $\begin{array}{c}\text { Best } \\
\text { candidates }\end{array}$ \\
\hline 0.0 & 0.2557 & 0.2823 & 0.2852 & $S_{3} \succ S_{2} \succ S_{1}$ & $S_{3}$ \\
0.1 & 0.2359 & 0.2605 & 0.2620 & $S_{3} \succ S_{2} \succ S_{1}$ & $S_{3}$ \\
0.2 & 0.2161 & 0.2386 & 0.2387 & $S_{3} \succ S_{2} \succ S_{1}$ & $S_{3}$ \\
0.3 & 0.1963 & 0.2167 & 0.2155 & $S_{2} \succ S_{3} \succ S_{1}$ & $S_{2}$ \\
0.4 & 0.1765 & 0.1948 & 0.1922 & $S_{2} \succ S_{3} \succ S_{1}$ & $S_{2}$ \\
0.5 & 0.1567 & 0.1729 & 0.1690 & $S_{2} \succ S_{3} \succ S_{1}$ & $S_{2}$ \\
0.6 & 0.1368 & 0.1510 & 0.1457 & $S_{2} \succ S_{3} \succ S_{1}$ & $S_{2}$ \\
0.7 & 0.1171 & 0.1291 & 0.1225 & $S_{2} \succ S_{3} \succ S_{1}$ & $S_{2}$ \\
0.8 & 0.0972 & 0.1072 & 0.0992 & $S_{2} \succ S_{1} \succ S_{3}$ & $S_{2}$ \\
0.9 & 0.0774 & 0.0854 & 0.0760 & $S_{2} \succ S_{1} \succ S_{3}$ & $S_{2}$ \\
1.0 & 0.0576 & 0.0634 & 0.0527 & $S_{2} \succ S_{1} \succ S_{3}$ & $S_{2}$ \\
\hline
\end{tabular}

$\tilde{E}_{2}=((0.462,0.609,0.868) ; 0.3,0.4)$,

$\tilde{E}_{3}=((0.451,0.624,0.857) ; 0.4,0.5)$.

- Step 8. In terms of Eqs. (16) and (25), the meanindex and standard deviation index of $\tilde{E}_{i}$ can be computed as follows:

$$
\begin{array}{ll}
M\left(\tilde{E}_{1}\right)=0.256, & D\left(\tilde{E}_{1}\right)=0.198, \\
M\left(\tilde{E}_{2}\right)=0.282, & D\left(\tilde{E}_{2}\right)=0.219, \\
M\left(\tilde{E}_{3}\right)=0.285, & V\left(\tilde{E}_{3}\right)=0.233 .
\end{array}
$$

- Step 9. Using Eq. (26), the ranking indices for the TIFNs $\tilde{E}_{i}(i=1,2,3)$ are obtained as follows:

$$
\begin{aligned}
& R_{\lambda}\left(\tilde{E}_{1}\right)=0.256-0.198 \lambda, \\
& R_{\lambda}\left(\tilde{E}_{2}\right)=0.282-0.219 \lambda, \\
& R_{\lambda}\left(\tilde{E}_{3}\right)=0.285-0.233 \lambda .
\end{aligned}
$$

- Step 10. The ranking indexes of each seller and the ranking order with different DM's preference information $\lambda$ are listed in Table 12.

From Table 12, if the DMs are optimistic (i.e., $0.5<\lambda \leq 1$ ), it can be seen that the best candidate is $S_{2}$. If the DMs are neutral (i.e., $\lambda=0.5$ ), the best candidate is still $S_{2}$. However, if the DMs are pessimistic (i.e., $0 \leq \lambda<0.5$ ), the best candidate changes from $S_{2}$ to $S_{3}$. Obviously, when the DM's risk preference value is different, the corresponding ranking order of alternatives is also not completely the same. Thus, the proposed method is rationality and flexibility.

\subsection{Comparison analysis with the existing methods}

To further illustrate the superiority of the method proposed in this paper, we adopt the method proposed by Wan et al. [27] to solve the above trustworthy service selection problem. The steps are given as follows:

- Step 1. Utilize Steps 1 and 2, which are described by Wan et al. [27], to derive the individual weight vectors 
of attributes $\mathbf{w}^{k}=\left(w_{1}^{k}, w_{2}^{k}, \cdots, w_{n}^{k}\right)^{T}(k=1,2,3,4)$ given by four DMs. The results are as follows:

$$
\begin{aligned}
& \mathbf{w}^{1}=(0.1605,0.5891,0.0482,0.1757,0.0265)^{T} \\
& \mathbf{w}^{2}=(0.0387,0.3680,0.1728,0.2248,0.2356)^{T}, \\
& \mathbf{w}^{3}=(0.3883,0.0277,0.2200,0.1657,0.1982)^{T}, \\
& \mathbf{w}^{4}=(0.2167,0.1738,0.3768,0.0198,0.2128)^{T} .
\end{aligned}
$$

- Step 2. Determine the weight vector of DMs $\mathbf{v}$ $=\left\{\nu_{1}, \nu_{2}, \cdots, \nu_{p}\right\}^{T}$ combining the evidence theory with Bayes approximation, which is proposed by Wan et al. [27]. Accordingly, we can have $\mathbf{v}=$ $\{0.2449,0.2490,0.2524,0.2537\}^{T}$. Thus, the collective attribute weight vector is obtained by Eq. (38) as follows:

$$
\mathbf{w}=(0.2012,0.2897,0.1941,0.1468,0.1682)^{T} .
$$

- Step 3. According to Steps 5-8, which are described by Wan et al. [27], the group utility values and individual regret values of each alternative can be calculated as follows:

$$
\begin{aligned}
& S\left(S_{1}\right)=0.5917, \quad S\left(S_{2}\right)=0.6508 \\
& S\left(S_{3}\right)=0.4972, \quad R\left(S_{1}\right)=0.1753 \\
& R\left(S_{2}\right)=0.1994, \quad S\left(S_{3}\right)=0.1221 .
\end{aligned}
$$

Thus, $S^{+}=0.4972, S^{-}=0.6508, R^{+}=0.1221$, and $R^{-}=0.1994$;

- Step 4. By Steps 9 and 10, which are defined in method [27], the closeness of each alternative to the ideal solution can be calculated as in Table 13;

- Step 5. Rank the alternatives in accordance with different coefficients of decision mechanism. The ranking order is summarized in Table 13.
From Table 13, we can obtain the same ranking order $\boldsymbol{S}_{\mathbf{2}} \succ \boldsymbol{S}_{\mathbf{1}} \succ \boldsymbol{S}_{\mathbf{3}}$ for three alternatives regardless of the parameter value of risk preference. The ranking order obtained by the extended VIKOR [27], $\boldsymbol{S}_{\mathbf{2}} \succ$ $\boldsymbol{S}_{\mathbf{1}} \succ \boldsymbol{S}_{\mathbf{3}}$, is just a special case of that obtained by the method proposed in this paper (i.e., the case of $0.8 \leq \lambda \leq 1$ as summarized in Table 13). Therefore, the result obtained by the proposed method in this paper meets the actual circs better.

We compare the proposed method with the method called "VIKOR method", introduced by Wan et al. [27]. There are some similarities and differences between the proposed method and VIKOR method. Their weights of attributes and DMs are completely unknown, and their idea of determining the weights of attributes and DMs is the same. However, the proposed method has some advantages:

(i) In determination of attribute weights stage, they both use entropy measure to determine the weights of attributes. The weight vector is based on the crisp relative closeness coefficient matrix directly derived from TIFNs matrix in this paper, whereas the weight vector of Wan et al. [27] is based on the crisp weighted possibility mean matrix defuzzified from the fuzzy decision matrix, in which the elements are the weighted possibility mean interval of the TIFNs. That is to say, the VIKOR method gets the crisp weighted possibility mean matrix through two transformations, whereas the proposed method gets the crisp relative closeness coefficient matrix through one transformation. As we all know, any transformation process may easily result in losses and distortions of information [32]. Thus, the decision information of the VIKOR method may be lost more than that of the proposed method in determining attribute weights;

(ii) In determination of DM weights stage, from

Table 13. The ranking order with different DM's preference information by using the extended VIKOR [27].

\begin{tabular}{cccccc}
\hline $\boldsymbol{\lambda}$ & $\boldsymbol{Q}\left(\boldsymbol{S}_{\mathbf{1}}\right)$ & $\boldsymbol{Q}\left(\boldsymbol{S}_{\mathbf{2}}\right)$ & $\boldsymbol{Q}\left(\boldsymbol{S}_{\mathbf{3}}\right)$ & $\begin{array}{c}\text { Ranking } \\
\text { orders }\end{array}$ & $\begin{array}{c}\text { Best } \\
\text { candidates }\end{array}$ \\
\hline 0.0 & 0.6885 & 1 & 0 & $S_{2} \succ S_{1} \succ S_{3}$ & $S_{2}$ \\
0.1 & 0.6812 & 1 & 0 & $S_{2} \succ S_{1} \succ S_{3}$ & $S_{2}$ \\
0.2 & 0.6739 & 1 & 0 & $S_{2} \succ S_{1} \succ S_{3}$ & $S_{2}$ \\
0.3 & 0.6666 & 1 & 0 & $S_{2} \succ S_{1} \succ S_{3}$ & $S_{2}$ \\
0.4 & 0.6593 & 1 & 0 & $S_{2} \succ S_{1} \succ S_{3}$ & $S_{2}$ \\
0.5 & 0.6520 & 1 & 0 & $S_{2} \succ S_{1} \succ S_{3}$ & $S_{2}$ \\
0.6 & 0.6447 & 1 & 0 & $S_{2} \succ S_{1} \succ S_{3}$ & $S_{2}$ \\
0.7 & 0.6374 & 1 & 0 & $S_{2} \succ S_{1} \succ S_{3}$ & $S_{2}$ \\
0.8 & 0.6301 & 1 & 0 & $S_{2} \succ S_{1} \succ S_{3}$ & $S_{2}$ \\
0.9 & 0.6228 & 1 & 0 & $S_{2} \succ S_{1} \succ S_{3}$ & $S_{2}$ \\
1.0 & 0.6155 & 1 & 0 & $S_{2} \succ S_{1} \succ S_{3}$ & $S_{2}$ \\
\hline
\end{tabular}


point of view of the consistency importance, their ideas are the same. Wan et al. [27] combines the evidence theory with Bayes approximation to give DM weights, but his calculation is complicated. Moreover, the evidence body of the VIKOR method is obtained also based on the possibility mean of TIFN, while this paper just needs to calculate the similarity of the individual decisions with respect to the average decisions. As compared with the VIKOR method, this method has relatively high accuracy with minor computing complexity.

Moreover, the proposed method can be extended to solve the MAGDM problems with other fuzzy numbers, such as trapezoidal intuitionistic fuzzy numbers, triangular fuzzy numbers, interval numbers, etc. Our future work is to apply the proposed method to different MAGDM problems, such as cloud service selection, credit risk analysis, etc.

\section{Conclusion}

With the rapid development of modern economy and the increasingly updated technology of internet, the real-world trustworthy service evaluation has become very complex. This paper firstly formulates the trustworthy service selection problem as a type of MAGDM with TIFNs. Thereby, a new method for MAGDM with TIFN and the completely unknown weights of DMs and attributes are proposed. The proposed method has some merits. First, we define mean-index, varianceindex, and standard deviation of TIFN, and we propose a new ranking method of TIFNs by taking into account the DMs' risk preference. Second, a new distance measure of TIFNs is defined and the corresponding proofs are given. Third, since the weights of attributes and DMs are directly derived from decision matrix with TIFNs, the information loss is minor in this MAGDM process. Then, this paper has less computing complexity. Finally, a comparison analysis is provided to illustrate the validity and reasonableness of the method proposed in this paper.

How to elicit the evaluation information of TIFNs is a critical issue before applying the proposed method. In near future, we will employ the expert system, evidence reasoning, fuzzy logic, behavior statistics, and data discovery as well as knowledge engineering to study this issue.

\section{Acknowledgment}

This work was partially supported by the National Natural Science Foundation of China (Nos. 61263018, 11461030, 61602219 and 71061006),Young scientists Training object of Jiangxi province (No.
20151442040081), the Natural Science Foundation of Jiangxi Province of China (No. 20161BAB201028), "Thirteen five" Programming Project of Jiangxi province Social Science (2016) (No. 16GL19), the Colleges Humanities Social Science Research Project of Jiangxi Province of China (No. GL162047) and the Science and Technology Project of Jiangxi province educational department of China (Nos. GJJ151601, GJJ150466 and GJJ150463).

\section{References}

1. Dubois, D. and Prade, H. "Operations on fuzzy numbers", International Journal of Systems Science, 9, pp. 626-631 (1978).

2. Merigó, J.M., Gil-Lafuente, A.M. and Yager, R.R. "An overview of fuzzy research with bibliometric indicators", Applied Soft Computing, 27, pp. 420-433 (2015).

3. Yager, R.R. "OWA aggregation over a continuous interval argument with applications to decision making", Systems, Man, and Cybernetics, Part B: Cybernetics, IEEE Transactions on, 34(5), pp. 1952-1963 (2004).

4. Wei, G.W. and Merigó, J.M. "Methods for strategic decision-making problems with immediate probabilities in intuitionistic fuzzy setting", Scientia Iranica, 19(6), pp. 1936-1946 (2012).

5. Dong, J.Y. and Wan, S.P. "Interval-valued trapezoidal intuitionistic fuzzy generalized aggregation operators and application to multi-attribute group decision making", Scientia Iranica, 22(6), pp. 2702-2715 (2015).

6. Xu, J., Wan, S.P. and Dong, J.Y. "Aggregating decision information into Atanassov's intuitionistic fuzzy numbers for heterogeneous multi-attribute group decision making", Applied Soft Computing, 41, pp. 331-351 (2016).

7. Atanassov, K.T. "Intuitionistic fuzzy sets", Fuzzy sets and Systems, 20(1), pp. 87-96 (1986).

8. Zeng, S.Z., Wang, Q.F., Merigó, J.M. and Pan, T.J. "Induced intuitionistic fuzzy ordered weighted averaging-weighted average operator and its application to business decision-making", Computer Science and Information Systems, 11(2), pp. 839-857 (2014).

9. Yu, D.J., Merigó, J.M. and Zhou, L.G. "Intervalvalued multiplicative intuitionistic fuzzy preference relations", International Journal of Fuzzy Systems, 15(4), pp. 412-422 (2013).

10. Xu, Y.J., Wang, H.M. and Merigó, J.M. "Intuitionistic Einstein fuzzy Choquet integral operators for multiple attribute decision making", Technological and Economic Development of Economy, 20(2), pp. 227-253 (2014).

11. Zadeh, L.A. "Fuzzy sets", Information and Control, 8(3), pp. 338-353 (1965).

12. Seikh, M.R., Nayak, P.K. and Pal, M. "Notes on triangular intuitionistic fuzzy numbers", International Journal of Mathematics in Operational Research, 5(4), pp. 446-465 (2013). 
13. Li, D.F. "A ratio ranking method of triangular intuitionistic fuzzy numbers and its application to MADM problems", Computers and Mathematics with Applications, 60, pp. 1557-1570 (2010).

14. Li, D.F., Nan J.X. and Zhang, M.J. "A ranking method of triangular intuitionistic fuzzy numbers and application to decision making", International Journal of Computational Intelligence Systems, 3(5), pp. 522530 (2010).

15. Zhang, M.J., Nan, J.X., Li, D.F. and Li, Y.X. "TOPSIS for MADM with triangular intuitionistic fuzzy numbers", Operations Research and Management Science, 21(5), pp. 96-101 (2012).

16. Wan, S.P., Li, D.F. and Rui, Z.F. "Possibility mean, variance and covariance of triangular intuitionistic fuzzy numbers", Journal of Intelligent and Fuzzy Systems, 24(4) pp. 847-858 (2013).

17. Wan, S.P. and Li, D.F. "Possibility mean and variance based method for multi-attribute decision making with triangular intuitionistic fuzzy numbers", Journal of Intelligent and Fuzzy Systems, 24(4), pp. 743-754 (2013).

18. Wan, S.P. "Multi-attribute decision making method based on possibility variance coefficient of triangular intuitionistic fuzzy numbers", International Journal of Uncertainty, Fuzziness and Knowledge-Based Systems, 21(2), pp. 223-243 (2013).

19. Wan, S.P. and Dong, J.Y. "Possibility method for triangular intuitionistic fuzzy multi-attribute group decision making with incomplete weight information", International Journal of Computational Intelligence Systems, 7(1), pp. 65-79 (2014).

20. Nan, J.X., Li, D.F. and Zhang, M.J. "A lexicographic method for matrix games with payoffs of triangular intuitionistic fuzzy numbers", International Journal of Computational Intelligence Systems, 3(3), pp. 280-289 (2010).

21. Zhang, X. and Liu, P. "Method for aggregating triangular fuzzy intuitionistic fuzzy information and its application to decision making", Technological and Economic Development of Economy, 2, pp. 280-290 (2010).

22. Robinson, J. and EC, H.A. "A search for the correlation coefficient of triangular and trapezoidal intuitionistic fuzzy sets for multiple attribute group decision making", In Mathematical Modelling and Scientific Computation, Springer Berlin Heidelberg, pp. 333-342 (2012).

23. Chen, Y. and Li, B. "Dynamic multi-attribute decision making model based on triangular intuitionistic fuzzy numbers", Scientia Iranica, 18(2), pp. 268-274 (2011).

24. Wang, J.Q., Nie, R., Zhang, H.Y. and Chen, X.H. "New operators on triangular intuitionistic fuzzy numbers and their applications in system fault analysis", Information Sciences, 251, pp. 79-95 (2013).
25. Yu, S. and $\mathrm{Xu}, \mathrm{Z}$. "Aggregation and decision making using intuitionistic multiplicative triangular fuzzy information", Journal of Systems Science and Systems Engineering, 23(1), pp. 20-38 (2014).

26. Wan, S.P. and Dong, J.Y. "Multi-attribute decision making based on triangular intuitionistic fuzzy number Choquet integral operator", Chinese Journal of Management Science, 22(3), pp. 121-129 (2014).

27. Wan, S.P., Wang, Q.Y. and Dong, J.Y. "The extended VIKOR method for multi-attribute group decision making with triangular intuitionistic fuzzy numbers", Knowledge-Based Systems, 52, pp. 65-77 (2013).

28. Grzegorzewski, P. "Distances between intuitionistic fuzzy sets and/or interval-valued fuzzy sets based on the Hausdorff metric", Fuzzy Sets and Systems, 148(2), pp. 319-328 (2004).

29. Wang, W. and Xin, X. "Distance measure between intuitionistic fuzzy sets", Pattern Recognition Letters, 26(13), pp. 2063-2069 (2005).

30. Qi, X., Liang, C. and Zhang, J. "Generalized crossentropy based group decision making with unknown expert and attribute weights under interval-valued intuitionistic fuzzy environment", Computers \& Industrial Engineering, 79, pp. 52-64 (2014).

31. Yue, Z. "A method for group decision-making based on determining weights of decision makers using TOPSIS", Applied Mathematical Modelling, 35(4), pp. 1926-1936 (2011).

32. Yue, Z. "An avoiding information loss approach to group decision making", Applied Mathematical Modelling, 37(1), pp. 112-126 (2013).

\section{Biographies}

Shuping Wan received a $\mathrm{PhD}$ degree in Control Theory and Control Engineer from Nankai University, Tianjin, China, in 2005. He is currently a Professor in College of Information Technology, Jiangxi University of Finance and Economics, China. He has contributed more than 80 journal articles to professional journals. His current research interests include decision analysis, fuzzy game theory, information fusion, and financial engineering.

Jun Xu received a $\mathrm{PhD}$ degree in Management Science and Engineering from Jiangxi University of Finance and Economics University, Nanchang, China in 2015. $\mathrm{He}$ is a Lecturer in College of Modern Economics \& Management of Jiangxi University of Finance and Economics. He has contributed more than 10 journal articles to professional journals. His research interests include decision analysis, fuzzy logic, and trusted computing. 